\title{
Machine learning as an enabler of qubit scalability
}

Intense efforts are underway to produce circuits that integrate a technologically relevant number of qubits. Although qubit control in most material systems is by now mature, device variability is one of the main bottlenecks in qubit scalability. How do we characterise and tune millions of qubits? Machine learning might hold the answer.

Not so long ago, the mastery required to encode and control a single qubit was reserved to a few experts. They were proud of their virtuosity. A student in pursuit of their PhD or an early-career researcher aiming at a Letter in Nature would be willing to persevere until they got the one working device needed for their experiments. To operate as qubits, quantum devices have to first be tuned, and researchers had to find the set of parameters that would encode a qubit and optimise its performance. But this single-minded single-device approach is not scalable for industrial quantum technologies.

Tuning procedures range from the super coarse to the very fine, with many characterisation steps in between required to validate previous steps and inform the next ones. Tuning is thus a time-consuming task, and when we are in possession of a winning set of parameters that allows us to operate and control a single qubit, we cannot simply apply it to another, nominally identical, qubit, because qubits are not sufficiently identical. The qubit devices that come closest are the atom-based ones, such as ion traps, but their control signals still need to be adaptive with time, introducing the requirement for tuning and stabilizing procedures. In many cases we are reaching the limits of our human ability to control these systems.

Realising the potential of quantum technologies will require circuits that integrate millions of qubits. Because identical qubit devices and/or perfectly stable control signals cannot be achieved, we need a tractable approach for the characterisation and tuning of large numbers of quantum devices. To be able to successfully tune and optimise qubit devices completely automatically, regardless of their variability, a big parameter space has to be explored, data has to be interpreted, patterns have to be recognised and decisions have to be made in real time. Machine learning can provide the key to speeding up, scaling up, and opening up new quantum technologies.

In the past few years, we have witnessed how breakthroughs in artificial intelligence, and in particular machine learning, are having a tremendous impact in science and technology. Although artificial intelligence has been available for a long time now, the availability of large data sets, the increasing computer power and the development of more powerful algorithms has given rise to a machine-learning revolution. In the past five years, groundbreaking achievements such as super-human image recognition, protein-structure solving and natural-language generation have been demonstrated. Powerful generative models and exquisite optimisation approaches are now giving us the opportunity to find overarching solutions for qubit control, taking qubit circuits to a new era.

Bringing together experts in machine learning and quantum technologies will enable us to address the question of how to make the most of current machine-learning advances for quantum device control. Two important trends in machine learning are the transition from classification to decision making and the transition from big data to small data. The amazing advances in the application of deep learning approaches were in large measure enabled by 
the availability of huge data sets. But for quantum devices the data can be scarce and costly to acquire. We are now seeing the rise of machine-learning techniques that can work with small amounts of data. These techniques can significantly reduce the number of measurements required for device characterization. Besides, simulations can produce useful training datasets for many qubit realisations. Machine-learning techniques can also speed up quantum device simulations.

These new machine-learning methods are also good at making choices. There is a trade-off between the time required for decision making versus the time invested in new measurements. In the limit of extremely fast measurements, it might be beneficial to choose the parameters randomly until the optimal combination is found. In the limit of slow measurements, an algorithm that evaluates the possibility of success for each set of parameters is paramount. Machine-learning approaches can speed up real-time measurements ${ }^{1}$, even choosing which measurements to perform next in order to maximize the information gain. ${ }^{2}$

\section{[H1] Tuning and characterisation of semiconductor quantum dot devices}

Because of the success of current semiconductor technologies, semiconductor quantum dot devices hold great promise for the production of complex quantum architectures. These devices can be lithographically defined and controlled electrically, and they exhibit promising qubit performances. As with other solid-state devices, however, small variations in their fabrication and defects in the material result in significant device variability. Although the fabrication of these devices has come a long way, this variability is far from being eliminated and it is even difficult to quantify.

One of my group's biggest achievements was to demonstrate that a machine-learning algorithm allows for the automatic tuning of these devices faster than human experts. ${ }^{3}$ This super-coarse tuning was an extremely complex task to automate, as device parameters define an 8-dimensional space and device characteristics vary non-monotonically and not always predictably with the control signals. Building a training set of measurements spanning the entire parameter space would have been a huge endeavour. In collaboration with DeepMind, we have overcome the complexity hurdle with classical machine-learning techniques, in particular making use of Gaussian processes. This demonstration has also allowed us to find a metric for device variability. We have demonstrated coarse and fine tuning using deep-learning approaches, ${ }^{4,5}$ because we were able to collect measurements spanning small parameter spaces for training datasets. Figure 1 illustrates the sequence of steps we are aiming to automate, going all the way from a 'virgin' semiconductor quantum chip to an array of optimised qubits.

\footnotetext{
${ }^{1}$ Bonato, C. et al. Nat. Nanotechnol. 11, 247 (2016)

${ }^{2}$ Lennon, D. T. et al. npj QI 5, 79 (2019)

${ }^{3}$ Moon, H. et al. Nat. Commun. 11, 4161 (2020)

${ }^{4}$ van Esbroeck, N. M. et al. New J. Phys. 22095003 (2020)

${ }^{5}$ Nguyen, V. et al. arXiv:2009.14825
} 


\section{[H1] Tuning and characterising other qubit devices}

The use of machine-learning approaches for quantum device tuning and characterisation in real time is also being established in other qubit realisations, such as superconducting qubits, nitrogen vacancy (NV) centres in diamond and ion traps. For example, an algorithm trained in real time was able to predict, from a set of measurements, the evolution of a superconducting qubit. ${ }^{6}$ Automatic detection of 31 individual nuclear spins was demonstrated using deep learning. ${ }^{7}$ Machine-learning techniques were also used to increase state readout fidelities in NV centres in diamond and trapped-ion qubits. ${ }^{8,9}$ In these pioneering works, it is becoming apparent that machine-learning algorithms are readily translatable between different qubit realisations, and that combined efforts would allow even faster progress.

\section{[H1] What's next}

The use of machine learning for quantum device control is transformative, and we will soon benefit from completely automated approaches that are applicable to a broad range of quantum devices. These demonstrations show a promising route towards the scalability of quantum processors. With efficient optimisation approaches, we might realise better qubit performances than those achieved so far. As the number of quantum devices increases, the automation and optimisation tasks we seek to address increase in complexity, and we shall have to push the frontiers of machine learning to be able to take the next steps forward. We are likely to need physics-aware algorithms and strategies to parallelize optimization tasks more efficiently. And what we will most certainly need is a well-cemented community of quantum-device and machine-learning experts working together to make it happen.

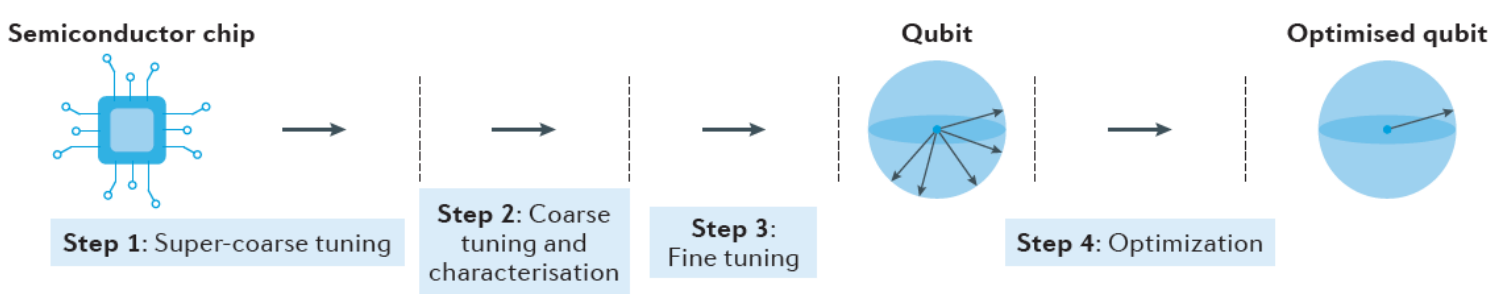

Figure 1. Qubit tuning and characterisation. A sequence of steps is needed to make a semiconductor chip into an array of optimised qubits; machine-learning approaches are being developed to fully automate this process.

\footnotetext{
${ }^{6}$ Flurin, E. et al. Phys. Rev. X 10, 011006 (2020)

7 Jung, K. et al. npj Quantum Information, 7, 41 (2021)

${ }^{8}$ Liu, Genyue et al. Mach. Learn.: Sci. Technol. 1015003 (2020)

${ }^{9}$ Ding, Zi-Han et al. Phys. Rev. Applied 12, 014038 (2019)
} 